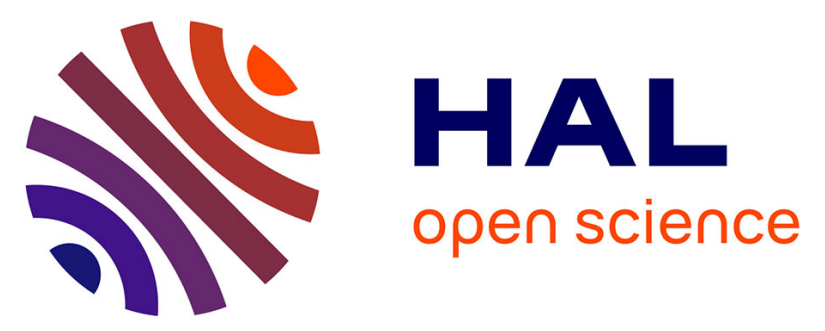

\title{
Dico: A Conceptual Model to Support the Design and Evaluation of Advanced Search Features for Exploratory Search
}

Emanuel Felipe Duarte, Edson Oliveira Jr., Filipe Roseiro Côgo, Roberto Pereira

\section{To cite this version:}

Emanuel Felipe Duarte, Edson Oliveira Jr., Filipe Roseiro Côgo, Roberto Pereira. Dico: A Conceptual Model to Support the Design and Evaluation of Advanced Search Features for Exploratory Search. 15th Human-Computer Interaction (INTERACT), Sep 2015, Bamberg, Germany. pp.87-104, 10.1007/978-3-319-22723-8_8. hal-01610794

\author{
HAL Id: hal-01610794 \\ https://hal.inria.fr/hal-01610794
}

Submitted on 5 Oct 2017

HAL is a multi-disciplinary open access archive for the deposit and dissemination of scientific research documents, whether they are published or not. The documents may come from teaching and research institutions in France or abroad, or from public or private research centers.
L'archive ouverte pluridisciplinaire HAL, est destinée au dépôt et à la diffusion de documents scientifiques de niveau recherche, publiés ou non, émanant des établissements d'enseignement et de recherche français ou étrangers, des laboratoires publics ou privés.

\section{(c)(1)}

Distributed under a Creative Commons Attribution| 4.0 International License 


\title{
Dico: A Conceptual Model to Support the Design and Evaluation of Advanced Search Features for Exploratory Search
}

\author{
Emanuel Felipe Duarte ${ }^{1}$, Edson OliveiraJr ${ }^{1}$, \\ Filipe Roseiro $\mathrm{Côgo}^{2}$, and Roberto Pereira ${ }^{3}$ \\ ${ }^{1}$ DIN, UEM, Maringá - PR, Brazil \\ contato@emanuelfelipe.net, edson@din.uem.br \\ ${ }^{2}$ DACOM, UTFPR, Campo Mourão - PR, Brazil \\ filiper@utfpr.edu.br \\ ${ }^{3}$ IC, UNICAMP, Campinas - SP, Brazil \\ rpereira@ic.unicamp.br
}

\begin{abstract}
The design of models and tools to support Exploratory Search acquires more importance as the amount of information on the Web grows. The use of advanced search features is a viable approach for query exploration during Exploratory Search. However, the usage of advanced search features remains relatively low since Web search engines became popular, partially because of design decisions that ignore the complex and flexible nature of search activities. In this paper, we introduce Dico: a conceptual model for advanced search features for Exploratory Search, presenting and evaluating a set of guidelines created to support designers and evaluators to design better advanced search features, promoting its usage. Results from an evaluation activity with prospective designers indicated participants were able to make sense of Dico's guidelines, suggesting the guidelines as a promising artifact to support the evaluation of search engines.
\end{abstract}

Keywords: Design and Evaluation Guidelines, Information Seeking, Exploratory Search, Advanced Search, Search User Interfaces.

\section{Introduction}

According to White and Roth [?], exploration is part of human nature and we aim to expand our knowledge through exploration. Continuous advances in information technology and the advent of the Web have revolutionized the way people interact with information, and the amount of information available to be explored has become abundant. However, the abundance of information became a significant problem in recent years as information consumes the attention of its recipients [?]. Therefore, White and Roth [?] express the need for systems capable of safeguarding users' attention through information filtering.

White, Kules and Bederson [?] discussed how search technologies available in 2005 already provided adequate support for users with well-defined information 
needs. However, the authors highlighted how these technologies lacked support for situations where users do not have the knowledge or the contextual awareness to formulate queries or navigate on complex information spaces. According to White et al. [?], current search technologies still provide insufficient support for this kind of activity, denominated Exploratory Search: an activity in which users perform a search with open and abstract goals and need to build knowledge about a particular subject. There is a growing need for models and tools to support Exploratory Search.

In this paper we introduce Dico (Latin for "say", "speak", "mean"): a conceptual model for advanced search features for Exploratory Search, presenting and evaluating a set of guidelines created to support designers and evaluators of Exploratory Search tools. These guidelines are intended to support the design and evaluation of advanced search features to provide new possibilities of exploration for people engaged in Exploratory Search activities, such as modifying, refining, restricting, or expanding a search query.

This paper is structured as follows: in Section 2 we briefly present and discuss the literature review; in Section 3 we present Dico, the methodology for creating the guidelines and the guidelines themselves; in Section 4 we present an evaluation of Dico's guidelines and discuss the main results; finally, in Section 5 we present the main conclusions and directions for future research.

\section{$2 \quad$ Literature Review}

According to White and Roth [?], most Web search engines operate by means of a classic query-response paradigm: users formulate a query and the search engine returns a collection of documents for the given query. However, this paradigm represents a narrow understanding about the Information Seeking process and the way people interact with information. Dico is grounded on Information Seeking models because a better understanding of the Information Seeking process can benefit the creation of models and tools for supporting Information Seeking activities, such as the Exploratory Search.

\subsection{Information Seeking}

As a research area, Information Seeking focus on Information Science and benefits from the advances of Computer Science, including Human-Computer Interaction (HCI). According to Marchionini [?], Information Seeking investigates the search for information centered on users and their activities. Attfield and Blandford [?] state that many Information Seeking models have been proposed with focus on temporal and behavioral aspects. However, several studies argue the users' iterative process is transversal to both temporal and behavioral aspects, and need to be considered [?], [?], [?].

Marchionini [?], for instance, describes the Information Seeking process in eight stages where iterations can occur, e.g. when the examination of the query 
results guides users to formulate a new query. According to Kuhlthau [?], cognitive processes and users' feelings throughout the activity also play an important role. Furthermore, the users' activity of finding meaning from information to extend their state of knowledge about a particular subject is considered in the author's Information Search Process. Negative feelings, such as uncertainty and anxiety, are common when there is little knowledge on the subject.

Bates' Berrypicking model [?] focus on the identification of new search opportunities, proposing a process in which users starts with only an open subject and shape their own path according to their evolving interests by navigating through different sources. The Berrypicking model emphasizes that: both information need and queries are constantly evolving; information is collected gradually and continuously; users perform a variety of search strategies according to the search topic and the proficiency with the involved technologies; and users navigate through various information sources to gradually and continuously build knowledge.

In a biological analogy, Pirolli [?] describes the Information Seeking process in terms of foraging. The author's Information Foraging theory explains how people explore information sources and decide whether to continue exploring the same region or identify a new and more "fruitful" area. This decision is motivated by a perceived information quality indicator named "information scent". In a vast information environment such as the Web, the perceived "information scent" of search results may define, or at least influence, the next steps of users.

To Dervin [?], people interact with an information system to build a "picture" or "model" of a domain. This process, called Sensemaking, can be described as "the deliberate effort to understand events" [?]. According to Zhang et al. [?], Sensemaking occurs when people face problems in unfamiliar situations where their current knowledge proves insufficient. Sensemaking plays an important role in search, specially when users' current knowledge on the topic is limited. Targeting for a holistic and high-level abstraction model about how people interact with information, Blandford and Attfield [?] created the Information Journey Framework. The Information Journey Framework suggests the design of tools to interact with information, such as Exploratory Search tools, should consider users' cognition process (i.e. the creation of mental models) and the iterative nature of the Information Seeking process, which may accommodate various techniques and interaction styles in addition to a variety of feelings from users.

\subsection{Exploratory Search}

White et al. [?] argue that Exploratory Search is an elusive concept, suggesting that every search is somewhat exploratory. Marchionini [?] develops a conceptual discussion and proposes a categorization with three types of search activities: "lookup", "learn" and "investigate", whereas Exploratory Search is concentrated on the last two. They are explained as follows:

- lookup: users already know the format of the information and how to find it, therefore well-defined queries are formulated generating accurate results. Example: a search for weather conditions; 
- learn: involves multiple iterations and a greater cognitive effort to handle and interpret the results. Possible goals are the acquisition of knowledge and skills, and the understanding of concepts. Example: a researcher seeking to learn what is quantum physics; and

- investigate: it also involves multiple iterations that may occur during long periods of time. This activity has higher-level goals, such as analysis, synthesis and evaluation. Example: a consumer interested in purchasing a television set investigating which model is the most appropriate for his needs.

According to White and Roth [?], Exploratory Search tools should "support querying and rapid query refinement" and "offer facets and metadata-based result filtering", among other features. In practical terms, these tools should support users to (re)formulate their queries, allowing them to constantly explore and filter the obtained results. In the literature, there are related works reporting results from investigations on Exploratory Search tools, such as Flamenco [?] and Relation Browser [?]. Although these tools feature important contributions, both Flamenco and Relation Browser are not suitable for the Web as a whole, they are limited to specific closed sets of documents because they need very carefully structured metadata.

In the sense of frameworks and design recommendations, there are related works such as Wilsons's [?] design recommendations and Campos and Silva's [?] framework. Aiming to propose a framework for search user interface features, Wilson [?] proposes four categories: (1) input; (2) control; (3) informational; and (4) personalisable, and twenty design recommendations distributed between these categories. The recommendations are succinct and direct in order to be easily applied. However, there is little room for discussion on human factors, and there is no clear connection with the domain of Exploratory Search. Campos and Silva [?], in turn, consider Exploratory Search directly and propose a framework with six dimensions that can be addressed in Exploratory Search tools: (1) documents quality; (2) information presentation; (3) time; (4) cognition and knowledge acquisition; (5) personalization; and (6) collaboration. Initially only time was directly explored with a history tool. Therefore, there is still need for subsequent studies to address other dimensions and their interrelations.

The aforementioned studies feature important contributions, however none of them considered the advanced search features' potential of exploration in the context of Exploratory Search activities. Morville and Callender [?] describe advanced search as a "clumsy" and rarely used complement, like Google Advanced Search ${ }^{1}$. To Russel-Rose and Tate [?], advanced search is a set of features that goes beyond what is provided by the conventional search user interface. Wilson [?] points out most advanced search features can be expressed as filters or operators in a query, as long as users memorize the proper syntax. However, Hearst [?] argues many users do not even understand how these operators work. Boolean algebra operators such as AND and OR can cause confusion.

Studies show only a small fraction of submitted queries makes use of advanced search features [?], [?], [?]. For instance, White and Morris [?] report that only

\footnotetext{
${ }^{1}$ http://www .google.com/advanced_search
} 
$1.1 \%$ of analysed queries contained advanced search features, and only $8.7 \%$ of users used at least one advanced search feature at some point. Summarizing the views of Hearst [?], Morville and Callender [?], Wilson [?] and Russell-Rose and Tate [?], we enumerate four major advanced search problems:

1. it displaces users from their context: memorization of filters and operators syntax is problematic for most users, therefore they need to access a separated "advanced search" page to make use of these features;

2. features are difficult to find: the link to access the separated "advanced search" page is usually concealed inside drop down menus or positioned in low visibility areas of the page;

3. features are presented in intimidating form: once users access the separated "advanced search" page, they usually find a long form full of fields with little or no help on how to fill them properly; and

4. the applied logic of the features can be misunderstood: it is common for people to have difficulties to understand the employed logic of some filters and operators, like the ones involving Boolean algebra.

As enumerated, advanced search features usage is relatively low due to several factors, some of them related to how these features are designed. However, because the use of advanced search features empowers users to explore new search possibilities, such as modifying, refining, restricting or expanding a search query, they are a plausible and viable approach to support Exploratory Search. Therefore, there is a need for studies that help to understand how to design better solutions that favour the use of advanced search features. Dico is intended to contribute in this direction and is presented in the next section.

\section{Dico}

Dico is a conceptual model for advanced search features to support Exploratory Search. Dico is composed by three principles derived from the four major problems pointed out in Section 2.2: (1) context, (2) presence and (3) simplicity, and has guidelines to promote the usage of advanced search features by addressing one or more of these principles. The conceptual model can be applied to any query-response paradigm search engine, such as Google ${ }^{2}$, Bing $^{3}$, Google Scholar $^{4}$ and Springer Link ${ }^{5}$. Fig. 1 illustrates Dico's composition and its application, which promotes the creation of Exploratory Search tools that favour the use of advanced search features. The main difference between Dico's guidelines and other examples of design recommendations, such as Nielsen's [?] heuristics or Wilson's [?] search user interface design recommendations, is the focus on promotion of advanced search features usage during Exploratory Search activities, an objective that may not be achieved without specific design recommendations such as Dico's guidelines.

\footnotetext{
2 http://www.google.com/

3 http://bing.com/

${ }^{4}$ http://scholar.google.com/

5 http://link.springer.com/
} 


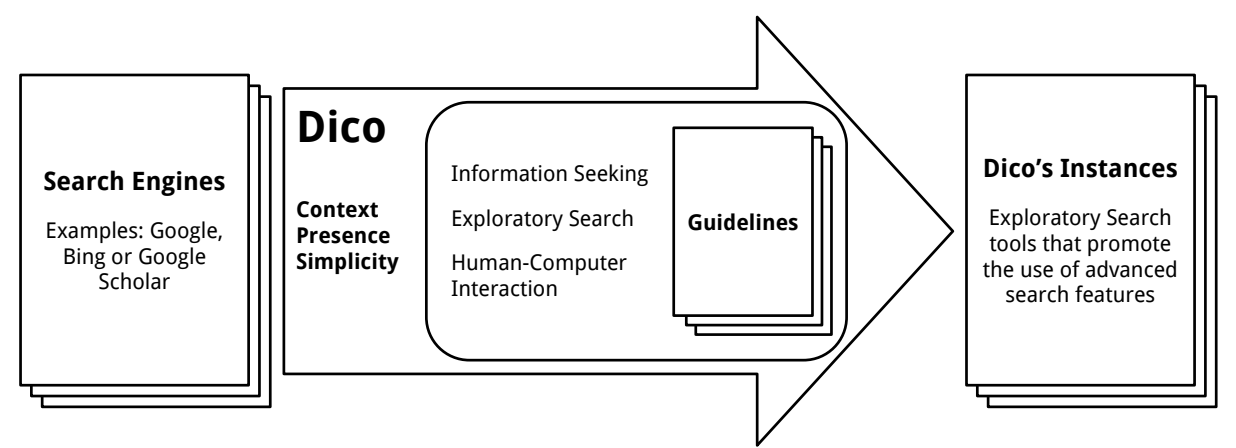

Fig. 1. Illustration of Dico's composition and its application.

\subsection{Methodology for Creating Dico's Guidelines}

Dico has three key principles that cover the major advanced search features problems identified in the literature: (1) context: provide features when and where they can be useful to users; (2) presence: make features easily found or perceivable by users; and (3) simplicity: present features without intimidating users and in formats they can understand what the feature is, what it does, and how to use it. Grounded on these principles, we carried out four steps to identify and propose guidelines for Dico. The methodology is illustrated in Fig. 2 and detailed in sequence:

- step 1: analyse how the studies discussed in the literature review are related to one or more of Dico's principles. If a study contributes to at least one principle it was a candidate to compose a new guideline or complement an already outlined one. Finally, compile a set of guidelines in which each one must be theoretically justified by at least one study. The guidelines' structure is inspired by Design Patterns [?], containing:

- name: presents an identification number and the guideline name. The number is for reference only and does not indicate importance or priority;

- description: presents a more detailed explanation of the guideline;

- theoretical foundation: describes the theoretical foundation used to support the guideline, which may be related studies on Information Seeking, Exploratory Search and HCI;

- category: relates the guideline to at least one of three Dico's principles, which are context, presence and simplicity; and

- example: presents and discusses an example of the guideline implementation (i.e., how it can be understood and put into practice).

- step 2: analyse the composed guidelines and complement the theoretical foundation with HCI studies. The considered studies are Nielsen's [?] heuristics, the communicability concept from Semiotic Engineering [?], and Weinschenk's [?] study on human aspects relevant to design decisions. These studies cover a wide range of factors, such as design questions and human factors (e.g., memory and cognition); 
- step 3: create an illustrated example of how to apply the guideline within a search user interface. Examples consist of an analysis of certain features of search user interfaces from search engines or academic tools. It is discussed how the example satisfies the guideline, or why it does not and what could be done to solve the problem; and

- step 4: evaluate the guidelines description and illustrated example with prospective designers. The guidelines were revisited based on the results from their usage and evaluation with a group of prospective designers. The evaluation details and results are presented in Section 4.

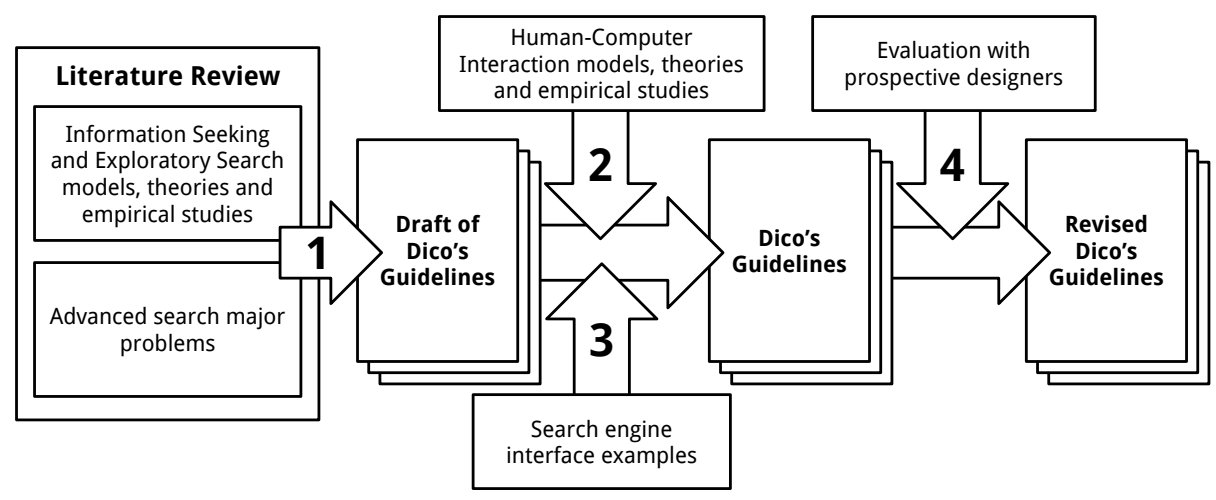

Fig. 2. Illustration of the methodological steps to create Dico's guidelines.

As a result of this process, we created a set of eight guidelines for Dico, which means we were able to approach Dico's principles with eight different perspectives inspired by our literature review. Due to space restrictions, we present only a summary of the guidelines. The summary contains the number, name and description for each guideline, and can be seen in Table 1. For demonstration purposes, guideline \#1 is presented in detail in Section 3.2. A document describing Dico and containing all the guidelines in their complete structure is available at http://din.uem.br/gsii/dico/.

\subsection{Guideline \#1: Users should be able to reformulate their query at any time in an easy way.}

- Description: the search user interface should always allow users to reformulate their query easily according to their evolving state of knowledge on the subject. The query reformulation can be in the format of modification, refinement, restriction, or expansion, and to do so the search field and the current query must always be available to users.

- Theoretical Foundation: as evidenced by Bates [?], Marchionini [?] and Blandford and Attfield [?], users go through an iterative process during a 
Table 1. Summary of Dico's guidelines.

\# Name and description

1 Users should be able to reformulate their query at any time in an easy way: the search user interface should always allow users to reformulate their query easily according to their evolving state of knowledge on the subject. The query reformulation can be in the format of modification, refinement, restriction, or expansion, and to do so the search field and the current query must always be available to users.

2 Advanced search features must be present in the main search page: advanced search features should not be limited to the fields of a separate and specific page, such features should be included in the main search page which users are already used to operate. More specifically, advanced search features should be included in the search results page, because it is there where they will possibly be more useful.

3 Users should be able to start their search in a conventional search user interface: to take advantage of advanced search features offered by the search engine, users should not be required to access an unfamiliar and possibly intimidating page. They should be able to start their search in a conventional search user interface they are already used to operate (e.g., the default home page of the selected search engine).

4 Advanced search features should complement information when applicable: when filters and operators relate to the presented information, interaction elements for advanced search features should be inserted in the search user interface next to the information or even contained in it. Such elements are intended to allow users to directly use the information of their interest to formulate and submit new queries without being moved from their context.

5 The interface should assist users to understand concepts and relationships present in the results: users should be assisted in forming a mental model of the researched topic, because this is an important step in the construction of knowledge in an Exploratory Search activity. The interface can provide a visual representation of the information space in which the main concepts and how they relate to the retrieved documents can be highlighted. Such relationships, for example, may be interactive so that users make further queries with related advanced search features.

6 Advanced search features must be presented in a manner that does not intimidate users: the search user interface should not have the format of a page with several fields to be filled or complex controls, it is necessary to provide search features that are as simples as possible according to the context. The advanced search features should be presented with simple controls, and the presentation of these features must be diluted along the search activity, with different features presented at different times as they become useful for a given context.

7 Users should be able to identify and manage advanced search features easily in the current query: the query must be processed so the features can be identified and presented in a format that highlights them individually. Presenting features individually also facilitates its management, like removing or editing a specific feature present in the query. When presented individually, the features may also receive an individual explanation in a language that makes sense to users, assisting them to associate syntax and functionality.

8 The search user interface should communicate technical information of advanced search features in a manner that makes sense to users: users must be able to understand the logical operation of advanced search features. For this, the visual and verbal communication used to display the features and related technical information must have a simple language that makes sense to users. 
search. Among other aspects, this process is characterized by query reformulation according to the new acquired knowledge. Therefore, it is critical the interface does not prevent or make it difficult for users to reformulate queries, because this is a recurring step during Exploratory Search activities. From the HCI perspective, this guideline can be related to Nielsen's third heuristic "user control and freedom", described by the author as "Users often choose system functions by mistake and will need a clearly marked 'emergency exit' to leave the unwanted state without having to go through an extended dialogue. Support undo and redo." Finally, considering the advanced search perspective, freedom and easiness to submit new queries can be incentives for users to explore the provided advanced search features.

- Category: context, presence and simplicity.

- Example: the search results page of the Brazilian e-commerce Submarino ${ }^{6}$, illustrated in Fig. 3 for the query "seo", makes use of features such as breadcrumbs, faceted navigation and visual highlight of the found terms. However, there are problems considering the scope of this guideline. Although the search field remains present on the Web page, it appears without the current query filled in, making it difficult to reformulate in case users wish to modify only a part of the query. The interface would be in compliance with the guideline if the search field remained filled after submitting a query.

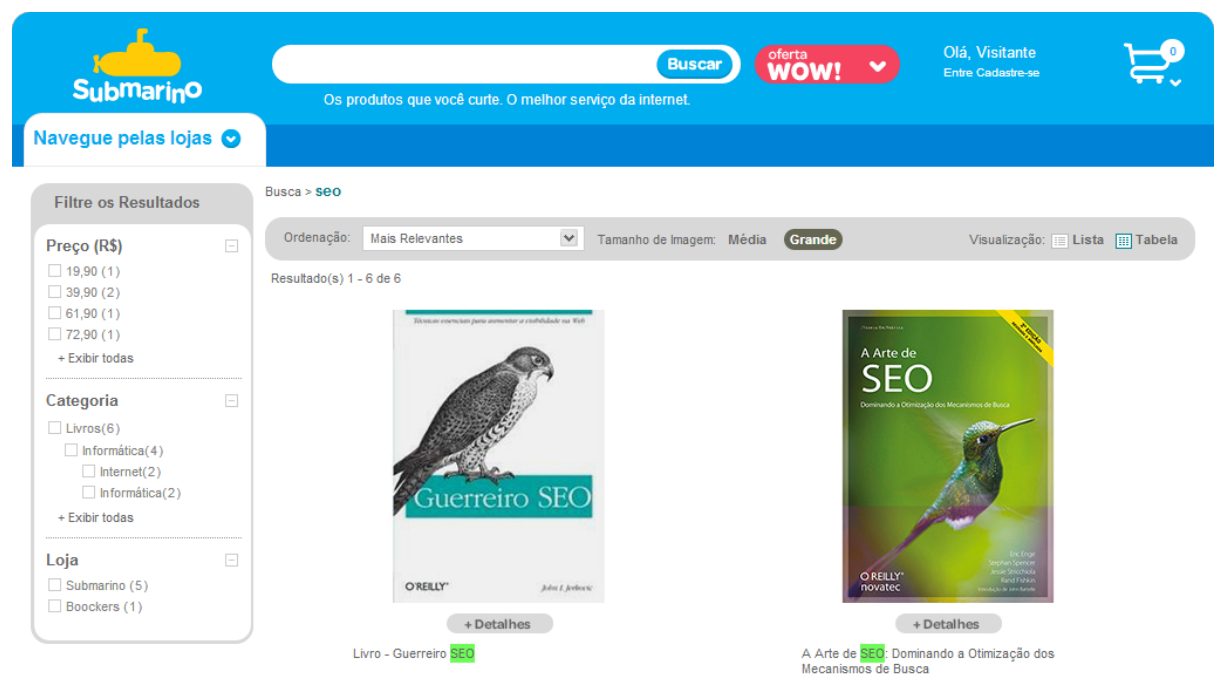

Fig. 3. Guideline \#1 example: lack of current query on the search field in the search results page of Submarino. Accessed 01 August 2014.

\footnotetext{
${ }^{6}$ http://www . submarino.com.br/
} 


\section{Evaluation}

To evaluate Dico's guidelines, we conducted a study to investigate whether prospective designers would make sense of the guidelines' descriptions and their illustrated examples, and whether they would find the guidelines useful to support their practical activities. First, participants carried out an inspection of two Web search engines using Dico's guidelines. This task was designed to introduce the guidelines to the participants, enabling them to subsequently evaluate the applied guidelines based on their experience with them. Both qualitative and quantitative data were collected and analysed, therefore we considered this a mixed evaluation. We used the Goal Question Metric (GQM) template [?] to summarize the nature of the study and the main aspects involved: we evaluated Dico's guidelines; for the purpose of assessing whether they make sense to designers who may apply them; with respect to description clarity and illustrated examples usefulness; from the viewpoint of designers using Dico's guidelines to inspect search engines; and in the context of Computer Science undergraduate students attending to a HCI course.

\subsection{Study Planning}

The study planning consisted of the activities described as follows.

Participant Selection: 15 Computer Science undergraduate students attending to a HCI course in the third semester were invited to participate. Participants were all males, with ages ranging from 18 to 31 years old. They were chosen for knowing basic principles of evaluation by inspection and heuristic evaluation, for being used to conduct Exploratory Search activities on the Web, and for being the target audience of Dico's guidelines: prospective designers who will probably work in the design and evaluation of computer systems. The study was applied as an HCI theoretical and practical activity, however it was up to the students to decide whether they want to anonymously supply their data to this study. A total of 14 students consented, and one of them had incoherent answers with the asked questions and was removed, resulting in a sample $N=13$. As the study involved human beings, the applicable ethical aspects were adequately addressed. Participation was voluntary, unpaid, with the possibility to leave the study at any time without justification, and all participants registered their consent.

Planning the Inspection of Search Engines with Dico: two search engines were selected to be inspected: Google (general purpose) and Google Scholar (scholarly literature), and participants were randomly assigned to inspect one of them. Individually, participants inspected whether the search engine was in compliance with each of Dico's guidelines. Participants did not receive any kind of prior training or information about Dico's guidelines beyond what is already presented in the guidelines. For data collection, we selected mandatory multiplechoice questions with three options: (1) "Yes"; (2) "No"; or (3) "N/A", followed 
by a required text field to justify the answer. This activity was considered sufficient to provide an initial experience with the application of Dico, qualifying the participants to later evaluate its guidelines directly.

Planning the Evaluation of Dico's Guidelines: participants evaluated the guidelines individually. For each guideline they evaluated the clarity of its description and the usefulness of its example. For data collection, we selected the format of an assertion for which participants select their degree of agreement using a five-point Likert scale. The first assertion is "The Guideline \#X is clear and can be easily understood", where $X$ is the guideline number. The possible answers were: (1) "Strongly agree"; (2) "Partially agree"; (3) "Indifferent"; (4) "Partially disagree"; and (5) "Strongly disagree". Participants have the option (not mandatory due to time constraints and possible fatigue effects) to justify their answer. The second statement is "The given example assists to understand the Guideline \#X". Again, participants must select an answer between the same degree of agreement options and optionally justify their answer.

Instrumentation: we created three ad hoc instruments for this study: (1) a document containing an overview of Dico's guidelines with their names and descriptions; (2) an online form for the inspection of search engines with Dico; and (3) an online form for the evaluation of Dico's guidelines. The first instrument contains a summary of all guidelines in the same page for a holistic view, because they can be viewed only one at a time in the online forms. The second instrument introduces and supports the search engine inspection with Dico, where each guideline along with the respective fields are presented in detail in separated pages. Finally, the third instrument introduces and presents Dico's guidelines evaluation, repeating the format in which each guideline along with the respective fields are presented in detail in separated pages.

\subsection{Operation}

Participation was in person during a HCI class inside a computer lab, and all participants had access to a computer and Internet. The first instrument was handed to each participant, and also contained an anonymous identification code and the search engine to be inspected. The other two instruments were given as URLs to be accessed. The participants were instructed to access them in order, starting with the form for the inspection of the search engines (second instrument), and then, after finishing filling it, accessing the form for the evaluation of Dico's guidelines (third instrument). Participants were instructed the purpose of the study was to obtain their opinion as prospective designers, and therefore there were no "correct" or "wrong" answers. The tasks were performed individually, without exchange of information between participants, and submitted in digital format. 


\subsection{Results Analysis and Interpretation}

For the search engine inspection with Dico, Fig. 4 shows the summary of responses for both Google and Google Scholar inspections. (In Fig. 4, "\#X" stands for compliance with guideline \#X according to participants' responses.) It can be noted that even though both search engines are from the same company, there is a clear difference in responses between them. This difference is positive for this study because both groups of participants had different experiences with the application of Dico's guidelines, enriching the results. As participants inspected the search engines with Dico, this experience properly qualified them to evaluate Dico's guidelines directly.

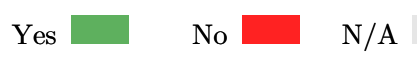

(a) Google

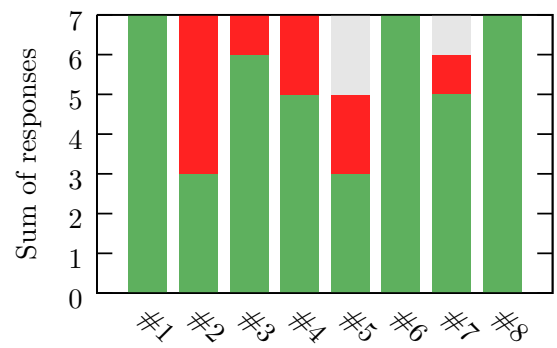

Dico's guidelines (b) Google Scholar

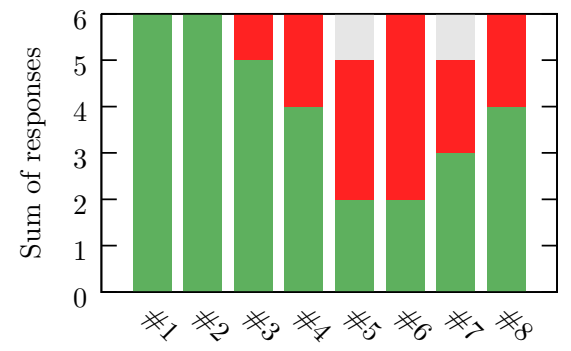

Dico's guidelines

Fig. 4. Summary of search engines inspection with Dico responses.

For Dico's guideline evaluation, Fig. 5 illustrate all responses (in the first activity participants 1, 3, 5, 7, 9, 11 and 13 inspected Google, and participants 2, 4, 6, 8, 10 and 12 inspected Google Scholar), and Fig. 6 a summary of the responses. (In Figs. 5 and 6, "\#XD" stands for the statement "The Guideline \#X is clear and can be easily understood" and "\#XE" for "The given example assists to understand the Guideline \#X".) Summarizing, there were 155 (74.52\%) responses for "Strongly agree", $30(14.42 \%)$ for "Partially agree" (summarizing $88.94 \%$ of agreeing responses), $12(5.77 \%)$ for "Indifferent", 8 (3.85\%) for "Partially disagree" and 3 (1.44\%) for "Strongly disagree" (summarizing 5.29\% of disagreeing responses). It can be noted participants 7 and 8 had together 16 of the 30 "Partially agree" responses. Aside from that, responses were relatively equally distributed among participants. The 11 disagreeing responses, for instance, were distributed among 7 participants, therefore we did not detect dramatic differences regarding individual participants. These results indicate participants approved Dico's guidelines descriptions and illustrated examples. Additionally, because participants were all prospective designers, it was 


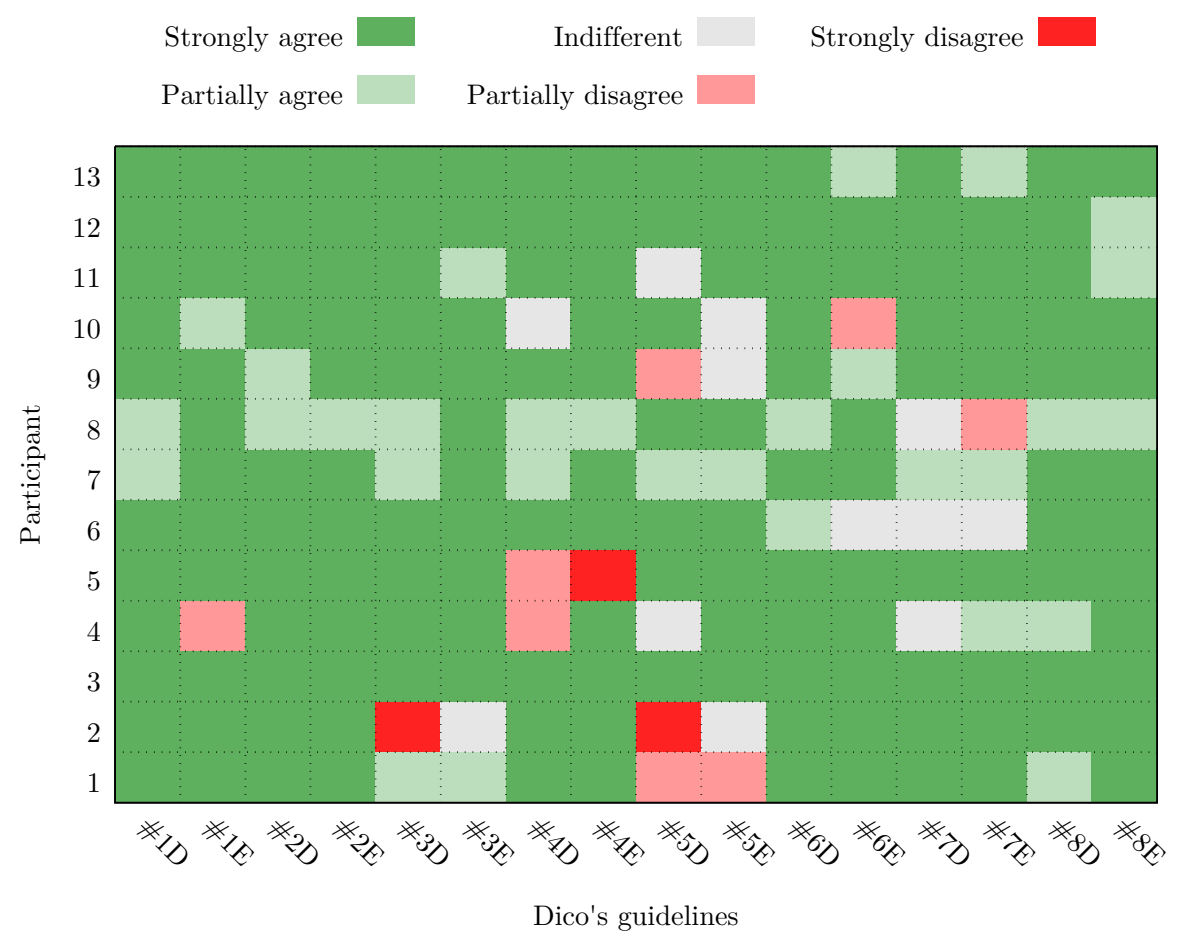

Fig. 5. Dico's guidelines evaluation responses.

expected them to experience some difficulty when using the guidelines for the first time. Only $5.29 \%$ of responses indicated difficulty, what is another indication the guidelines were well received and understood. However, a more detailed analysis regarding individual guidelines and differences between the responses of participants who inspected Google and Google Scholar can provide more specific results and highlight opportunities for improvement.

For guidelines \#2 and \#8, all the participants strongly or partially agreed their descriptions are clear and can be easily understood and the illustrated example assists to understand the guideline. One participant, for instance, justified his "Strongly agree" choice for guideline \#8 as "Very easily understood." for description clarity and "Clear and easily understood example." for illustrated example usefulness - all translations of participants answers were made by the authors. Therefore, guidelines \#2 and \#8 were considered useful and well elaborated by all the participants. It can be noted that among participants who inspected Google there were 3 "Partially agree" responses, while for those who inspected Google Scholar there were 6, indicating these two guidelines were better understood when compared against Google. This result may suffer influence from users' previous experience with the search engine and from specific design 


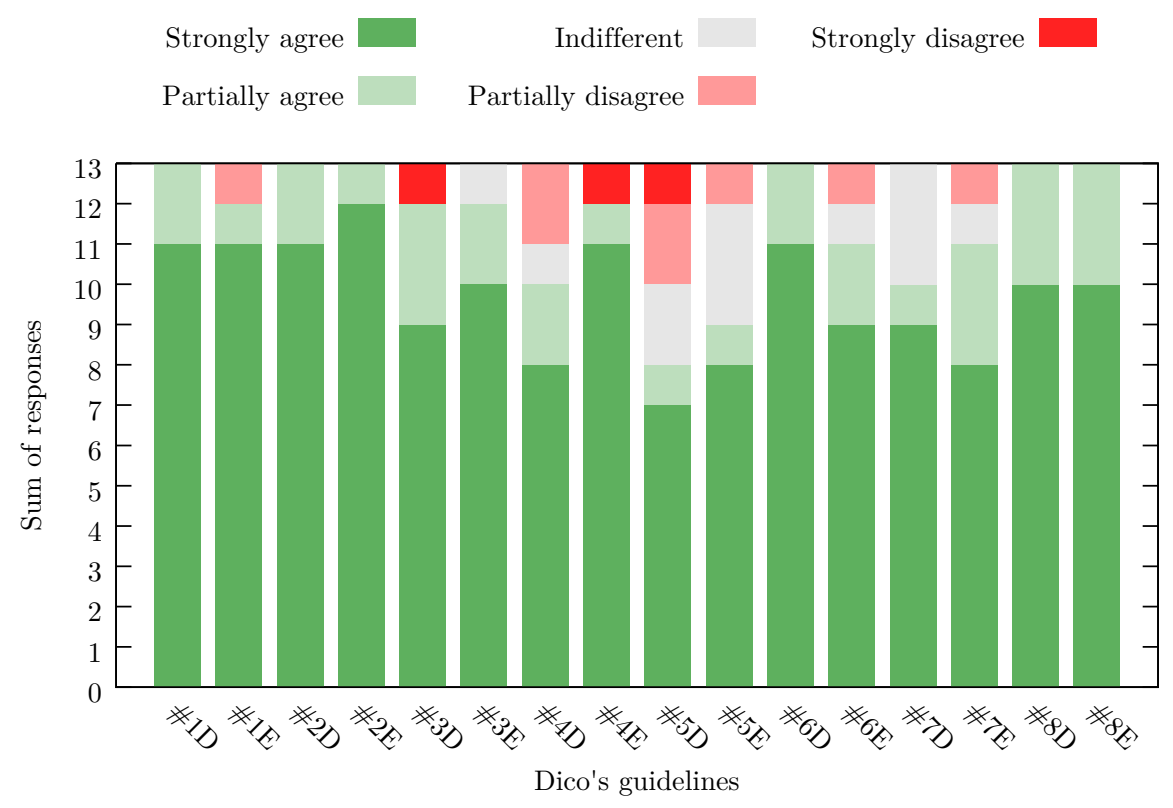

Fig. 6. Summary of Dico's guidelines evaluation responses.

decisions. A new study with a larger sample of participants and search engines may offer results of statistical significance to verify these hypothesis.

For guidelines \#1, \#3, \#6 and \#7, each received one "Partially disagree" or "Strongly disagree" response for description clarity (guideline \#3) or illustrated example usefulness (guidelines $\# 1, \# 6$ and \#7). One participant, for instance, justified his "Strongly disagree" choice for guideline \#3 description clarity as "I found it very difficult to understand what the guideline means." It can be noted only "Strongly agree" or "Partially agree" responses were provided by participants who evaluated Google, while all occurrences of "Indifferent", "Partially disagree" and "Strongly disagree" responses were from participants who inspected Google Scholar. Guideline \#7 description clarity had 3 neutral responses, however the participants did not justify their choices for these responses. Even though we did not identify evident issues with guidelines \#1, \#3, $\# 6$ and \#7, the presence of indifferent and disagreeing responses motivated a review of them. Again, it is possible these guidelines are better understood when compared against Google, however further studies are needed for conclusions.

Finally, for guidelines \#4 and \#5, there were occurrences of more than one "Partially disagree" or "Strongly disagree" responses, being two for guideline \#4 and three for guideline \#5, both on description clarity. It can be noted most "Indifferent", "Partially disagree" and "Strongly disagree" responses come from participants who inspected Google, contrary to the other six guidelines in which all these type of responses came from participants who inspected Google 
Scholar. One participant who inspected Google justified his "Indifferent" choice for guideline \#5 description clarity as "I was unable to apply the guideline in the search engine." Other, who inspected Google Scholar, justified his "Strongly disagree" for the same item as "No, the guideline is very difficult to understand, I was unable to extract the information the guideline means." Even though the majority of participants still provided "Strongly agree" or "Partially agree" responses for both guidelines, we identified the recurrence of other responses as a result of some participants having difficulty to understand the guidelines \#4 and \#5 abstraction level, therefore they were improved by a review with focus on description simplicity.

The difference between responses for participants who evaluated Google and Google Scholar indicate designers may understand some of Dico's guidelines differently in different contexts, such as specific domain search engines. It is possible some of Dico's guidelines are prone to make more sense in the context of some search engines than in others. This possibility should be considered and further analysed in future studies. In general, even guidelines with problematic feedback, like \#4 and \#5, still made sense to the majority of participants, indicating the prospective designers who participated in this study succeeded in making sense of Dico's guidelines and showing the guidelines as a promising artifact to support the evaluation of existing Web search engines.

\subsection{Validity Evaluation}

Following, we present the potential threats to the study's validity we anticipated, and the efforts taken to minimize them.

Conclusion Validity: the study sample size $(N=13)$ does not provide results with statistical significance, and participants applied Dico to only two different search engines. However, our objective was to obtain feedback from prospective designers because they are Dico's target audience. Even though a larger sample of participants and search engines is needed for results with statistical significance, the study sample size was considered enough to obtain initial evidence Dico's guidelines descriptions and illustrated examples made sense to the participants. Therefore, sample size was not considered a threat.

Construct Validity: we were very careful for participants to properly understand what should be done in the activity. Instructions for every step were explained clearly and succinctly before the study, the tasks performed by participants were coherent with their qualification and educational level and they were allowed to ask for help at any time if they had questions about how to proceed. To ensure the same treatment for all the participants, questions about how to proceed in the study were answered to the whole class. No participant showed significant difficulty in understanding and carrying out the activity. Therefore, the threat of participants not understanding what should be done was considered under control, not affecting the results. 
Internal Validity: we anticipated the following two threats:

1. fatigue effects: participants took between $1 \mathrm{~h}$ and $1 \mathrm{~h} 30 \mathrm{~m}$ to complete the study, and it required a considerable cognitive workload from them. However, the participants are used to perform similar activities during the $1 \mathrm{~h} 40 \mathrm{~m}$ duration HCI classes in which the study took place, and they could have abandoned the study at any time if they became uncomfortable with it. Therefore, the threat of participants becoming fatigued was considered under control, not affecting the results; and

2. influence among participants: because we requested participants to carry out the activity individually, and no significant interaction between them was observed, the threat of participants influencing each others' responses was considered under control, not affecting the results.

External Validity: the selection of prospective designers as participants does not guarantee experienced designers would show similar responses. However, we intentionally selected prospective designers to evaluate Dico because they are its target audience. Because low experienced prospective designers were able to make sense of Dico's guidelines descriptions and illustrated examples, it is reasonable to expect experienced designers to also be able to make sense of them, possibly even better than prospective designers. Therefore, we did not consider the selection of prospective designers a threat.

\section{Conclusions}

Current search engines still lack support for Exploratory Search. Therefore, there is a need for models and tools to support users in Exploratory Search activities. Even though the use of advanced search features is a plausible approach for Exploratory Search tools because it empowers users with new exploration possibilities, currently the usage of such features is relatively low.

To promote the use of advanced search features we introduced Dico. Dico is composed of three principles and eight guidelines to address the current major problems of advanced search. Inspired by Design Patterns, each guideline has: identification number and name; description; theoretical foundation on Information Seeking, Exploratory Search and HCI; category according to the three principles; and illustrated example demonstrating how it can be applied.

We evaluated Dico's guidelines and provided evidence prospective designers were able to make sense of them when inspecting existing Web search engines. Even though most responses evidenced no problems, the few who did also provided useful insights to further improve some of Dico's guidelines. For further studies regarding Dico, we consider:

- evaluate Dico's guidelines with a larger sample and more search engines to obtain results of statistical significance and possibly new insights on how different contexts affect the ability to make sense of Dico's guidelines; 
- analyse designers using Dico's guidelines to identify problems in search engines and propose design solutions to analyse how differently people may apply the guidelines and, by evaluating the solutions, whether Dico's guidelines are serving their purpose in assisting the design better tools; and

- instantiate Dico as an Exploratory Search tool based on a search engine and evaluate it both qualitatively and quantitatively to analyse aspects such as how participants feel about using the tool, and measure the usage of advanced search features to compare with data from the literature.

Acknowledgements. This study was partially funded by Coordenação de Aperfeiçoamento de Pessoal de Nível Superior (CAPES). We would like to dedicate this work to Professor Sérgio R. P. da Silva who is dearly missed. 